\title{
Anisotropic scaling of ripple morphologies on high-fluenc sputtered silicon
}

\author{
Adrian Keller, ${ }^{1, *}$ Rodolfo Cuerno, ${ }^{2}$ Stefan Facsko, ${ }^{1}$ and Wolfhard Möller ${ }^{1}$ \\ ${ }^{1}$ Institute of Ion Beam Physics and Materials Research, Forschungszentrum Dresden Rossendorf, \\ P.O. Box 510119, 01314 Dresden, Germany \\ ${ }^{2}$ Departamento de Matemáticas and Grupo Interdisciplinar de Sistemas Complejos (GISC), Universidad Carlos III de Madrid, \\ Avenida de la Universidad 30, E 28911 Leganés, Spain
}

\begin{abstract}
The evolution of $\mathrm{Si}(100)$ surfaces has been studied during oblique high fluenc ion sputtering by means of atomic force microscopy. The observed surface morphology is dominated by nanoscale ripples and kinetic roughening at small and large lateral scales, respectively. The large scale morphology exhibits anisotropic scaling at high fluence with different roughness exponents $\alpha_{n}=0.76 \pm 0.04$ and $\alpha_{p}=0.41 \pm 0.04$ in the direc tions normal and parallel to the incident ion beam, respectively. Comparison to the predictions of single fiel and two fiel ("hydrodynamic") models of ion erosion suggests the relevance of nonlinearities that are not considered in the simpler anisotropic Kuramoto Sivashinsky equation.
\end{abstract}

DOI: 10.1103/PhysRevB.79.115437

PACS number(s): 81.16.Rf, 68.37.Ps, 79.20.Rf, 81.65.Cf

\section{INTRODUCTION}

It is known since the 1960 s that oblique low and medium energy ion sputtering may induce a self-organized process at the irradiated surface that leads to the formation of periodic ripple structures with periodicities in the submicron range. ${ }^{1,2}$ These ripple patterns have been found on all kinds of amorphous as well as crystalline materials such as insulators, ${ }^{1}$ semiconductors, ${ }^{3}$ and metals. ${ }^{2}$ The periodicity of the ripples was found to scale with ion energy and can be tuned from few tens to several hundreds of nanometers. ${ }^{4}$

Nowadays, the ion-induced formation of nanopatterns gains new interest. To some extent, this interest is caused by the availability of new techniques for the precise characterization of the eroded surfaces. There are several in situ and ex situ studies that investigate the ripple formation by means of light scattering, ${ }^{5} \mathrm{x}$-ray scattering, ${ }^{6}$ and scanning probe techniques. ${ }^{7,8}$ In addition, ion-induced ripple patterns become also interesting for certain technological applications, e.g., in microelectronic device fabrication. ${ }^{9}$ Moreover, nanorippled substrates were also found to significantl influenc the magnetic ${ }^{10}$ and optical ${ }^{11}$ properties of metallic thin films The formation process of the ripples itself, however, is still not completely understood.

In 1988, Bradley and Harper ${ }^{12}(\mathrm{BH})$ developed a continuum model to describe the formation and evolution of ripple patterns during ion erosion. It was already shown by Sigmund, ${ }^{13}$ under the assumption of a Gaussian distribution of the energy deposited by the impinging ion, that the local erosion rate of a rough surface under ion bombardment is higher in depressions than on elevations. This curvature dependence of the erosion rate induces an instability of the surface against periodic disturbances that causes an amplifi cation of the initial roughness spectrum. In the presence of a competing smoothing process such as surface self-diffusion, however, wavelength selection is observed associated with the dynamic dominance of a single Fourier mode of the surface height. ${ }^{12}$ Hence, the formation of periodic structures on the surface results directly from a competition between roughening due to removal of surface material and smooth- ing by thermal or ion-induced diffusion ${ }^{14}$ or viscous flo in the case of amorphous surfaces. ${ }^{6,15}$

The linear continuum equation derived by $\mathrm{BH}$ is able to reproduce some of the main experimentally observed features of the pattern formation such as the orientation of the ripples with respect to the ion beam and the exponential growth of the ripple amplitude. For long sputtering times, however, certain experimental observations such as the saturation of the ripple amplitude cannot be explained within the framework of the $\mathrm{BH}$ model. This disagreement was attributed to a growing influenc of nonlinear terms that dominate the morphology at later times. Hence, nonlinear continuum models generalizing the Kuramoto-Sivashinsky (KS) equation to anisotropic systems have been derived for describing the evolution of the ripple structures. ${ }^{4,16}$ In the early-time regime, these models behave like the linear $\mathrm{BH}$ equation. At a certain transition time $t_{c}$, however, the nonlinear terms start to control the evolution of the surface. ${ }^{17}$ In this nonlinear regime, the amplitude of the ripples saturates as observed experimentally. ${ }^{18}$ For longer sputter times, however, a transition to isotropic kinetic roughening occurs. ${ }^{17,19}$ Although such a transition has been found in some experiments, ${ }^{20}$ other studies report persistence of the ripple pattern at high fluences ${ }^{21}$ More recent nonlinear models focus on this pattern stabilization at long times ${ }^{22}$ or the phenomenon of nonuniform transverse ripple motion and ripple coarsening. ${ }^{23,24}$

In face of the complexity of formulating continuum models to describe these phenomena starting from firs principles, an alternative approach consists in exploiting the occurrence of collective phenomena, such as scaling properties of height fluctuations Thus, a standard way to identify the relevant continuum model when kinetic roughening takes place is the analysis of the dynamic scaling behavior of the surface morphology. ${ }^{25}$ To this end, the surface can be characterized in space and time by the global interface width $W(L, t)$ $=\left\langle[h(\vec{r}, t)\langle h(\vec{r}, t)\rangle]^{2}\right\rangle^{1 / 2}$ calculated over the whole system size $L$. Here, $h(\vec{r}, t)$ is the surface height function and the angular brackets denote spatial averaging. Since physical systems typically have rather large system sizes, $W(L, t)$ is hardly accessible in experiments. Therefore, the so-called lo- 
cal interface width $w(l, t)$ is usually evaluated with $l \ll L$ being the size of an observation window. In the case of FamilyVicsek dynamic scaling, ${ }^{25,26}$ the local and the global interface widths should both satisfy the dynamic scaling an satz

$$
w(l, t)=t^{\beta} f\left(l / t^{1 / z}\right),
$$

with the scaling function

$$
f(u) \sim \begin{cases}\text { const. } & \text { if } u \gg 1, \\ u^{\alpha} & \text { if } u \ll 1 .\end{cases}
$$

Here, $\alpha$ is called the roughness exponent, $z$ the dynamic exponent, and $\beta=\alpha / z$ the growth exponent. Based on these dynamic scaling exponents, the system can be assigned to a certain universality class and, therefore, to a certain type of continuum equation. ${ }^{25}$

With such an aim, we have investigated the evolution and the dynamic scaling behavior of ripple morphologies on $\mathrm{Si}$ surfaces during sub-keV ion sputtering. At low fluences the surface morphology is dominated by a well-ordered ripple pattern. At higher fluences however, this pattern is overlayed by larger corrugations which dominate the surface morphology. Similarly, dominant long-wavelength corrugations have been also reported at large fluence for normal-incidence bombardment of Si targets. ${ }^{27}$ At high fluences the largedistance morphology exhibits a scaling anisotropy with different roughness exponents in the direction normal and parallel to the projection of the incident ion beam, respectively. This result is at variance with previous observations of isotropic kinetic roughening in experiments of this type and cannot be reconciled with current knowledge on the scaling properties of the anisotropic KS equation. ${ }^{17,19}$ As a consequence, the potential relevance of dispersive nonlinearities ${ }^{4,28}$ and/or those appearing in two-fiel models $^{23,24}$ seems naturally suggested.

\section{EXPERIMENT AND ANALYSIS}

The experiments were performed in an ultrahigh vacuum chamber with a base pressure of about $10^{8}$ mbar. A standard Kaufman-type ion source was used to bombard commercially available epipolished $\mathrm{Si}(100)$ wafers with a native surface oxide with $500 \mathrm{eV} \mathrm{Ar}^{+}$ions. The angle of incidence was fixe at $\theta=67^{\circ}$ with respect to the surface normal. The applicability of Sigmund's Gaussian approximation under these experimental conditions has been verifie by TRIDYN (Ref. 29) calculations. Due to the impact of the ions, the firs few nanometers of the crystalline Si surface get nearly immediately amorphized ${ }^{30}$ resulting in an amorphous top layer ${ }^{31}$ of few nanometers thickness. The sample temperature during the experiments was always below $180^{\circ} \mathrm{C}$ so that no recrystallization of the amorphous layer is expected. The applied ion flu ranged from $3.5 \times 10^{14}$ to $3.5 \times 10^{15} \mathrm{~cm}^{2} \mathrm{~s}^{1}$. Within this range, no influenc of the applied flu on the surface morphology could be observed. After sputtering with different fluence $\Phi$ in the range from $1 \times 10^{16}$ to 1 $\times 10^{20} \mathrm{~cm}^{2}$, the surface topography of the samples was analyzed ex situ by means of atomic force microscopy (AFM).
In order to study the scaling behavior of the surface morphology during its evolution, the one-dimensional power spectral density or structure factor has been calculated from lateral cuts of the AFM images. The structure factor is define as $S(k, t)=\langle\hat{h}(k, t) \hat{h}(k, t)\rangle$, with the Fourier transform $\hat{h}(k, t)$ of the surface height of the one-dimensional cut $h(r, t)$ and the spatial frequency $k$. In the case of Family-Vicsek scaling, it can be expressed by the relation ${ }^{25}$

$$
S(k, t)=k^{(2 \alpha+1)} s\left(k t^{1 / z}\right),
$$

with the scaling function

$$
s(u) \sim \begin{cases}\text { const. } & \text { if } u \gg 1, \\ u^{2 \alpha+1} & \text { if } u \ll 1 .\end{cases}
$$

Anisotropic surfaces such as the ones studied in the present work may exhibit an even more complex dynamic scaling behavior than isotropic ones since the anisotropy of the surface is also reflecte in the lateral correlations of the surface roughness. ${ }^{28,32}$ Thus, considering for simplicity the stationary state, the height-difference correlation function or, equivalently, the local roughness scales with different exponents in the directions normal $(n)$ and parallel $(p)$ to the projected direction of the ion beam, $w\left(l_{n, p}\right) \sim l_{n, p}^{\alpha_{n, p}}$, where $l_{n, p}$ refers to one-dimensional (1D) cuts being taken in the corresponding directions. Similarly, the one-dimensional structure factor $S_{n, p}\left(k_{n, p}\right)$ must be evaluated independently in both directions behaving as

$$
\left.S\left(k_{n, p}\right) \sim k_{n, p}^{\left(2 \widetilde{\alpha}_{n, p}+2\right.} \chi_{n, p}\right),
$$

where

$$
\tilde{\alpha}_{n, p}=\alpha_{n, p} \frac{1 \chi_{n, p}}{2}
$$

are two different roughness exponents and $\chi_{n}=\alpha_{n} / \alpha_{p}$ and $\chi_{p}=1 / \chi_{n}$ are called the anisotropy exponents. Thus, the roughness exponents measured in real and reciprocal spaces differ although, being related through Eqs. (5) and (6), there are only two independent ones. ${ }^{28}$ Note that, by substituting Eq. (6) into Eq. (5), the latter reads finall

$$
S\left(k_{n, p}\right) \sim k_{n, p}^{\left(2 \alpha_{n, p}+1\right)} .
$$

In our case, we have determined the real-space normal and parallel roughness exponents $\alpha_{n, p}$ by evaluating the onedimensional structure factor curves at the highest fluence according to Eq. (7). In order to minimize the influenc of measurement artifacts, the $S_{n, p}\left(k_{n, p}\right)$ curves have been averaged over six AFM images taken at different positions on the surfaces of up to fiv identically treated samples.

\section{RESULTS}

Figure 1 shows AFM images of the morphology after sputtering with four different fluences At a fluenc of $\Phi$ $=5 \times 10^{16} \mathrm{~cm}^{2}$ [Fig. 1(a)], shallow ripples appear with a wavelength $\lambda \simeq 24 \mathrm{~nm}$ that are oriented normal to the projected direction of the ion beam. These ripples are relatively short and not well ordered. With increasing fluence however, 

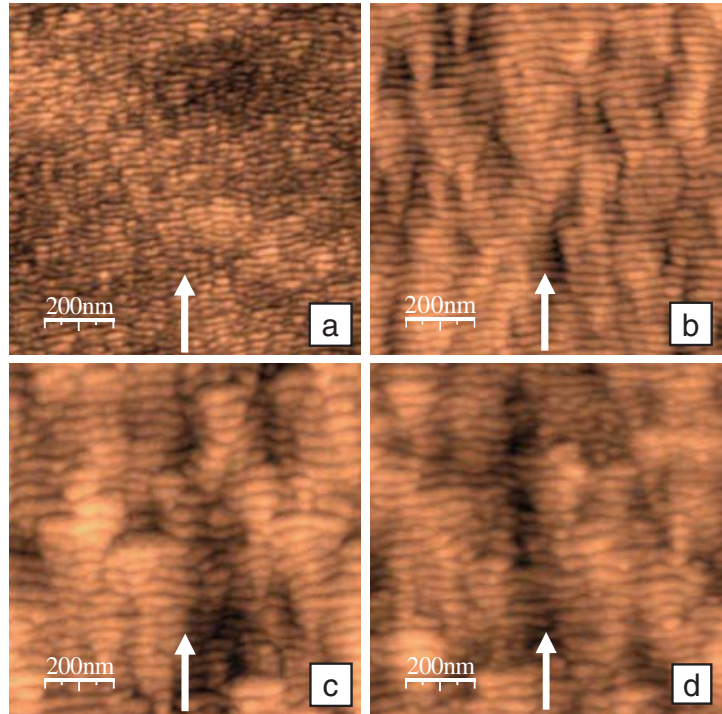

FIG. 1. (Color online) AFM images of $\mathrm{Si}(100)$ after sputtering with $500 \mathrm{eV} \mathrm{Ar}^{+}$ions at fluenc (a) $\Phi=5 \times 10^{16}$, (b) $5 \times 10^{17}$, (c) $2 \times 10^{19}$, and (d) $1 \times 10^{20} \mathrm{~cm}^{2}$. The white arrows indicate the di rection of the incident ion beam. Intensity scales are (a) 4 , (b) 8 , and [(c) and (d) $] 11 \mathrm{~nm}$.

they become longer and more homogeneous and also their wavelength increases [Fig. 1(b), $\Phi=5 \times 10^{17} \mathrm{~cm}^{2}$ ]. At this stage, larger corrugations form and overlay the ripple pattern. These corrugations become more pronounced with fluenc until they dominate the surface morphology [Figs. 1(c) and 1(d)]. At much larger lateral scales, these corrugations even show a kind of periodicity. ${ }^{33}$ In this regime, coarsening stops and the ripple wavelength is found to saturate at a fluenc $\Phi \simeq 10^{19} \mathrm{~cm}^{2}$.

In Fig. 2(a), the structure factor curves in the direction normal to the ion beam, $S_{n}\left(k_{n}\right)$, are depicted for different fluences Toward large values of $k_{n}$, the $S_{n}$ curves all coincide. The slope $m$ (in the log-log plot) of the curves in this regime is about 4 , corresponding to a roughness exponent of 1.5. At small $k_{n}$ values, however, $S_{n}\left(k_{n}\right)$ increases with fluenc and a second scaling regime develops. The extrapolated value of the roughness exponent in this long-range scaling regime is $\alpha_{n}=0.76 \pm 0.04$. For high fluence $\Phi$ $>10^{19} \mathrm{~cm}^{2}$, the $S_{n}$ curves coincide also at low $k_{n}$. However, at the lowest $k_{n}$ values, a slight rounding of the structure factor curves is observed even for the highest fluenc applied. This might indicate that the large-scale morphology has not fully saturated yet.

The structure factor $S_{p}\left(k_{p}\right)$ calculated in the direction parallel to the ion beam is given in Fig. 2(b). In this direction, for $\Phi \geq 5 \times 10^{16} \mathrm{~cm}^{2}$, a peak appears at the spatial frequency $k_{p}^{*}$ corresponding to the wavelength $\lambda$ of the ripple pattern. For $k_{p} \gg k_{p}^{*}$, again a slope $m=4$ is observed. With increasing fluence the ripples coarsen and the position of the peak is shifting to smaller $k_{p}$ values. As in the direction normal to the ion beam, the structure factor increases with flu ence for $k_{p} \ll k_{p}^{*}$ and again shows a power-law behavior at high fluences Here, the roughness exponent was determined to be $\alpha_{p}=0.41 \pm 0.04$. As in the $n$ direction, albeit less pronounced, the $S_{p}$ curves of the highest fluence exhibit a slight rounding at the lowest $k_{p}$ values.
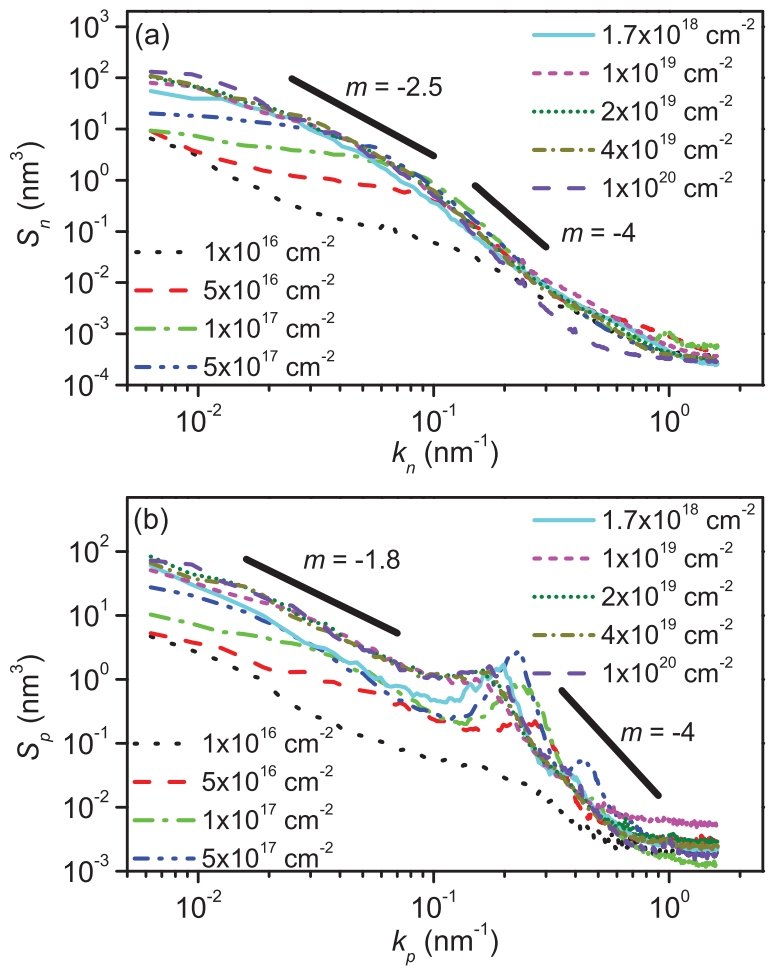

FIG. 2. (Color online) Structure factors $S_{n, p}\left(k_{n, p}\right)$ in the direction (a) normal and (b) parallel to the ion beam for $\mathrm{Si}(100)$ sputtered at different fluences Straight solid lines correspond to $S_{n, p} \sim k_{n, p}^{m}$.

Note that the apparent power-law behavior of the $S_{n, p}$ curves at low $k_{n, p}$ for the lowest fluence given in Figs. 2(a) and 2(b) still resembles the virgin Si substrate. With increasing fluence however, the influenc of the original substrate morphology vanishes.

From Eqs. (3) and (4) it follows that plotting the rescaled structure factor $S(k, t) k^{(2 \alpha+1)}$ versus $k t^{1 / z}$ for the different times, all curves should collapse into a single one. In this way, the dynamic exponents $z_{n, p}$ can be measured by using the previously determined roughness exponents $\alpha_{n, p}$. Figure 3 shows the collapsed structure factor curves for low flu ences. In both directions, a good convergence is obtained for $\alpha_{n, p}=1.5$ and $z_{n, p}=4.5$. The low- $k_{n, p}$ slope of the collapsed structure factor curves should be given by $m=2 \alpha_{n, p}+1=4$. This behavior is reasonably well confirme as can be seen from the straight solid lines in Fig. 3.

For high fluences Figs. 4(a) and 4(b) show the collapsed structure factor curves for low $k_{n, p}$ values. In the $n$ direction, again a good convergence is obtained. In the $p$ direction, however, it is less perfect. This can be attributed to the fact that the $p$ direction is also the slow-scan direction of the AFM and, therefore, rather sensitive to sampling-related artifacts especially at long wavelengths. Nevertheless, the obtained collapse is still reasonable as is indicated again by the straight black lines in Figs. 4(a) and 4(b) that exhibit slopes of $m=2 \alpha_{n}+1=2.52$ and $m=2 \alpha_{p}+1=1.82$, respectively. After the system reaches its stationary state, one expects $\beta$ $=\alpha_{n, p} / z_{n, p}=0$ and, therefore, very large effective $z_{n, p}$ values. The curves shown in Fig. 4, however, were obtained for $z_{n}$ $=8$ and $z_{p}=9$. Considering the uncertainties due to sampling- 

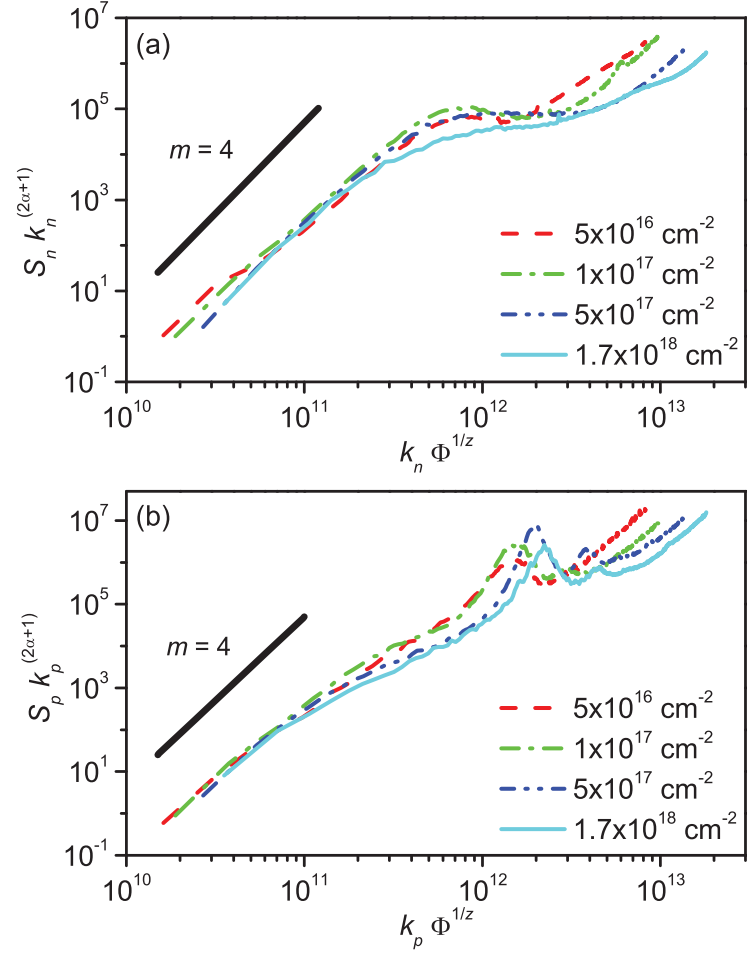

FIG. 3. (Color online) Collapsed structure factor curves at low fluence in the direction (a) normal and (b) parallel to the ion beam, respectively. The collapses were obtained using $\alpha_{n, p}=1.5$ and $z_{n, p}$ $=4.5$. Straight solid lines have a slope $m$.

induced artifacts especially in the $p$ direction, the difference between these two values is probably within the error margins of the data collapses and not caused by a real anisotropy. Although the high-fluenc $z_{n, p}$ values are by a factor of 2 larger than the ones obtained at low fluences they are still of comparable magnitude. This supports the interpretation that the system is not completely saturated yet as already indicated by the rounding of $S_{n, p}(k, t)$ at small $k$ for the highest fluence (cf. Fig. 2). At full saturation, one would expect the small $k$ data in Fig. 2 to better follow the corresponding solid lines, which thus currently provide extrapolations of the expected asymptotic behavior. However, no fina conclusions on this issue can be drawn from the current data set. For this, one would need different experimental techniques that probe the surface at larger lateral scale such as surface-sensitive $\mathrm{x}$-ray scattering.

The evolution of the ripple amplitude $a$, define as the half of the average peak-to-peak height of the ripples, is shown in Fig. 5(a). In the low-fluenc regime, the amplitude $a$ is increasing from initially $0.4 \mathrm{~nm}$ to a maximum value of about $0.8 \mathrm{~nm}$ at $\Phi \simeq 5 \times 10^{17} \mathrm{~cm}^{2}$. For higher fluences the amplitude decreases again and finall saturates at a value of $a^{\text {sat }} \simeq 0.6 \mathrm{~nm}$. A similar overshooting before saturation has already been observed for the local and global interface widths in previous experiments under normal ion incidence ${ }^{34}$ and simulations of the anisotropic KS equation, ${ }^{17}$ respectively. Because of the small value of the ripple amplitude, shadowing effects that, e.g., become important at high flu ences in the case of medium energy ion sputtering ${ }^{35}$ can be excluded for the current experimental conditions. ${ }^{36}$ Figure
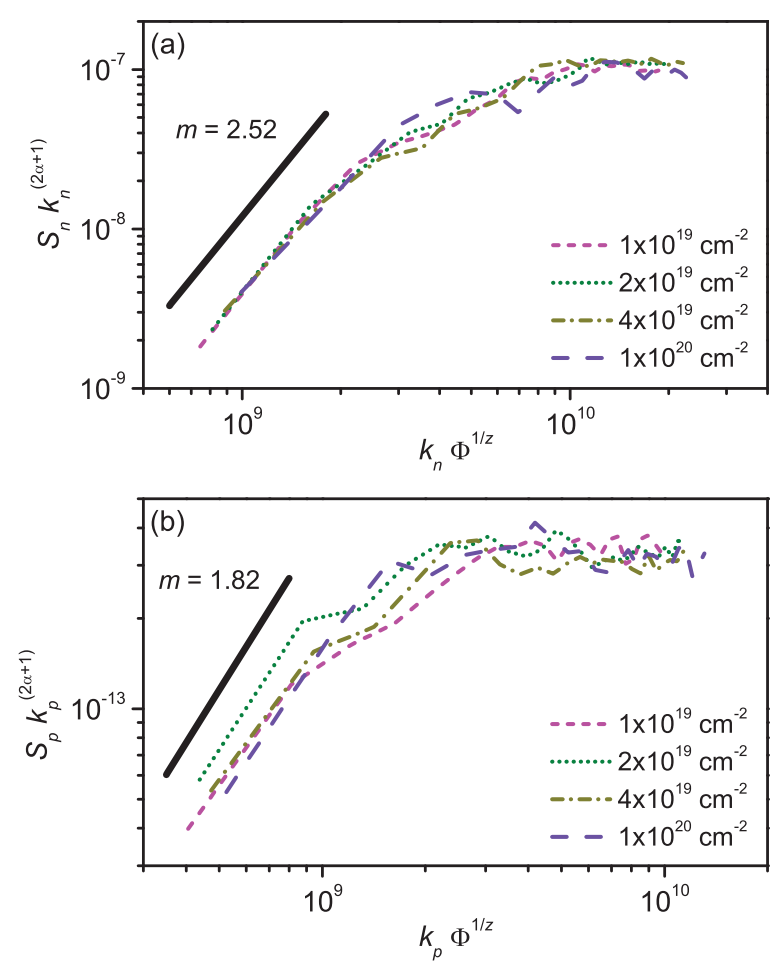

FIG. 4. (Color online) Collapsed structure factor curves for $k_{n, p} \ll k_{p}^{*}$ at high fluence in the direction (a) normal and (b) parallel to the ion beam, respectively. The collapses were obtained using $\alpha_{n}=0.76, z_{n}=8, \alpha_{p}=0.41$, and $z_{p}=9$. Straight solid lines have a slope $m$.

5 (b) depicts the evolution of the ripple wavelength $\lambda$ measured from the main peak of the parallel structure factor. As indicated by the straight line, logarithmic coarsening is ob-
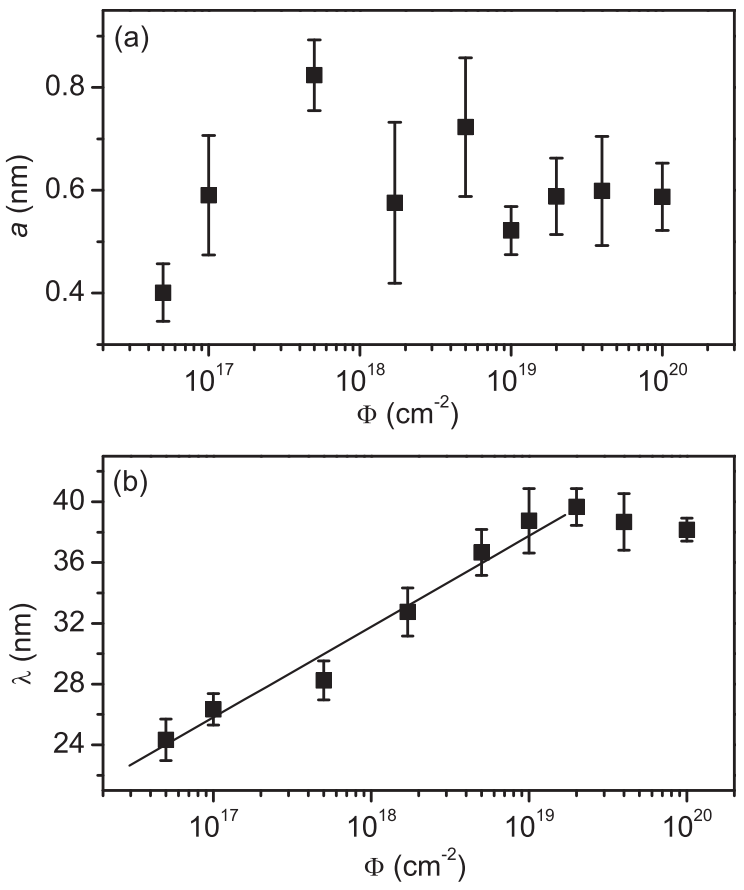

FIG. 5. Evolution of (a) ripple amplitude $a$ and (b) ripple wave length $\lambda$. Solid line in (b) represents a logarithmic fit 
served until the wavelength saturates at a fluenc of $\Phi$ $\simeq 10^{19} \mathrm{~cm}^{2}$.

\section{DISCUSSION}

\section{A. Short-range scaling}

The observed peak in the structure factor $S_{p}$ in the direction parallel to the ion beam with the 4 slope at large $k_{p}$ values [cf. Fig. 2(b)] indicates the presence of a KS type instability in this direction. ${ }^{37}$ The anisotropic KS equation for the surface height is given by ${ }^{16}$

$\partial_{t} h=\gamma \partial_{x} h+\nu_{n} \partial_{x}^{2} h+\nu_{p} \partial_{y}^{2} h+\frac{\zeta_{n}}{2}\left(\partial_{x} h\right)^{2}+\frac{\zeta_{p}}{2}\left(\partial_{y} h\right)^{2} \quad D \nabla^{4} h+\eta$

Here, $\gamma$ is the lateral velocity of the ripples, $D$ is the thermal or ion-induced diffusion, ${ }^{14}$ and $\eta$ is a noise term that accounts for the stochastic nature of the sputtering process. The surface instability is incorporated by the linear coefficient $\nu_{n, p}$, with at least one of them being negative. The coeffi cients of the Kardar-Parisi-Zhang ${ }^{38}$ (KPZ) nonlinearities, $\zeta_{n, p}$, incorporate the dependence of the local erosion velocity on the surface slopes.

The orientation of the ripples with respect to the incident ion beam is determined by the signs of the linear coeffi cients: the wave vector of the observed ripple structure is parallel to the direction with the smallest negative $\nu{ }^{12,16}$ Therefore, for the present experiment $\nu_{p}<\nu_{n}$. Concerning the value of $\nu_{n}$, we can distinguish three cases, as follows from the exact form of $S(k, t)$ for the linearization of Eq. (8), that are accurate for small time and length scales. Note that in principle the very short-distance behavior of Eq. (8) is dominated by the $D \nabla^{4} h$ term, inducing the scaling behavior of the linear molecular-beam epitaxy (MBE) equation, whose exponent values in $(1+1)$ dimensions $\operatorname{are}^{25} \alpha_{\mathrm{MBE}}=3 / 2$ and $z_{\mathrm{MBE}}=4$.

(i) $\nu_{n}>0$. For a sizeable and positive $\nu_{n}$ the scaling behavior should cross over from linear MBE to EdwardsWilkinson (EW) type, ${ }^{25}$ the power characterizing the $S\left(k_{n}\right)$ decay with $k_{n}$ clearly departing from the 4 value at intermediate time and length scales.

(ii) $\left|\nu_{n}\right| \approx 0$. For $\left|\nu_{n}\right|$ close to zero, no transition to EW scaling would occur and the 1D linear MBE scaling would hold even for intermediate times.

(iii) $0>\nu_{n}>\nu_{p}$. A negative $\nu_{n}$ value would introduce a second instability in the $n$ direction. Although the instability in the $p$ direction would overcome this second instability very soon, the corresponding structure factor $S_{n}$ would exhibit a local maximum at $k_{n}^{*}=\sqrt{\left|\nu_{n}\right| / 2 D}$. However, for $\left|\nu_{n}\right|$ $\ll\left|\nu_{p}\right|$, the local maximum might be too small to be recognized in the structure factor curve.

The experimental $S_{n}$ curves shown in Fig. 2(a) do not exhibit a local maximum. The determined low-fluenc behavior for the $n$ direction $S\left(k_{n}\right) \sim k_{n}{ }^{4}$ as induced by the $D \nabla^{4} h$ term holds even at the highest applied fluenc of $\Phi$ $=1 \times 10^{20} \mathrm{~cm}^{2}$. This indicates that the second case with $\left|\nu_{n}\right| \approx 0$ applies most likely. Physically, ${ }^{39}$ we thus expect the condition $0>\nu_{n} \gg \nu_{p}$ to apply in the current experiments.

\section{B. Long-range scaling}

Although the long-range scaling behavior of Eq. (8) is still unexplored, two different long-time scaling regimes are expected depending on the relative signs of the nonlinear coefficient $\zeta_{n}$ and $\zeta_{p}$. For $\zeta_{n} \zeta_{p}>0$, an algebraic scaling behavior similar to the KPZ equation ${ }^{38}$ is expected whereas $\zeta_{n} \zeta_{p}<0$ might result in isotropic logarithmic scaling. ${ }^{4,16}$ However, it was shown that for $\zeta_{n} \zeta_{p}<0$ rotated ripples, socalled cancellation modes appear at long times and dominate the surface morphology. ${ }^{17,19}$

For the current experimental conditions, one would expect $^{4} \zeta_{n} \zeta_{p}<0$. However, even for the highest applied flu ence of $\Phi=1 \times 10^{20} \mathrm{~cm}^{2}$, neither the appearance of rotated ripple structures nor isotropic logarithmic scaling is observed. On the contrary, for $\Phi \geq 10^{19} \mathrm{~cm}^{2}$, the morphology exhibits anisotropic algebraic scaling at large length scales with $\alpha_{n}=0.76 \pm 0.04$ and $\alpha_{p}=0.41 \pm 0.04$. Although these exponents agree fairly well with the exponents of the isotropic $\mathrm{KS}^{40}$ (early-time regime) and the isotropic KPZ equation, ${ }^{25}$ respectively, the strong degree of anisotropy in the system rather suggests that this similarity is just coincidental.

In Eq. (8), the only term breaking the $x \rightarrow x$ symmetry is the one with parameter $\gamma$. However, in order to fully describe the nonlinear evolution of ion-sputtered surfaces to the same order in powers of $h$ and space derivatives, Makeev et al. ${ }^{4}$ derived the following general equation:

$$
\begin{aligned}
\partial_{t} h= & \gamma \partial_{x} h+\nu_{n} \partial_{x}^{2} h+\nu_{p} \partial_{y}^{2} h+\Omega_{1} \partial_{x}^{3} h+\Omega_{2} \partial_{x} \partial_{y}^{2} h+\xi_{n}\left(\partial_{x} h\right)\left(\partial_{x}^{2} h\right) \\
& +\xi_{p}\left(\partial_{x} h\right)\left(\partial_{y}^{2} h\right)+\frac{\zeta_{n}}{2}\left(\partial_{x} h\right)^{2}+\frac{\zeta_{p}}{2}\left(\partial_{y} h\right)^{2} \quad D \nabla^{4} h+\eta
\end{aligned}
$$

where we note in particular the appearance of dispersive nonlinearities with coefficient $\xi_{n, p}$. This general equation has a rather complex parameter space and different scaling behaviors may be expected depending on the nonlinear coefficients Although to date the dynamic scaling exponents of Eq. (9) still have to be clarified we have additional information from a different particular case of this equation. Specifi cally, Schmittmann et al. ${ }^{28}$ performed a renormalizationgroup calculation for the (linearly stable) case in which $\nu_{n, p}>0$, while $\gamma=\Omega_{1}=\Omega_{2}=\zeta_{n, p}=0$. Remarkably, they fin parameter regimes in which the stationary state is characterized by strong anisotropy, in the sense that $\chi_{n, p} \neq 1$ and thus $\alpha_{n} \neq \alpha_{p}$.

Another experimental observation that can be explained neither with the KS nor with the general Eq. (9) is the coarsening of the ripple wavelength. Nevertheless, the recent twofiel or "hydrodynamic" model developed by Muñoz-García et al., ${ }^{23,24,41}$ in which the height fiel is coupled to a density of material that diffuses at the surface, is able to show ripple coarsening at intermediate times. The resulting equation is similar to Eq. (9) but with additional conserved KPZ nonlinearities of the form $\sum_{i, j} x, y \zeta_{i j}^{(2)} \partial_{i}^{2}\left(\partial_{j} h\right)^{2}$, namely,

$$
\begin{aligned}
\partial_{t} h= & \gamma \partial_{x} h+\nu_{n} \partial_{x}^{2} h+\nu_{p} \partial_{y}^{2} h \quad D \nabla^{4} h+\Omega_{1} \partial_{x}^{3} h+\Omega_{2} \partial_{x} \partial_{y}^{2} h \\
+ & \xi_{n}\left(\partial_{x} h\right)\left(\partial_{x}^{2} h\right)+\xi_{p}\left(\partial_{x} h\right)\left(\partial_{y}^{2} h\right)+\frac{\zeta_{n}}{2}\left(\partial_{x} h\right)^{2}+\frac{\zeta_{p}}{2}\left(\partial_{y} h\right)^{2} \\
& \sum_{x, y} \zeta_{i j}^{(2)} \partial_{i}^{2}\left(\partial_{j} h\right)^{2}+\eta
\end{aligned}
$$


In this model, the ripple wavelength remains constant at short times as in the linear BH equation. Due to the high flu of the Kaufman ion source used, this linear regime cannot be accessed in the current experiments. ${ }^{33}$ Then, at intermediate times, the $\zeta_{i j}^{(2)}$ nonlinearities are seen to induce the coarsening of the ripples. At long times, however, the nonconserved KPZ nonlinearities $\zeta_{j}$ [such as those in Eqs. (8) and (9)] become dominant and coarsening stops. ${ }^{24,41}$ In this regime, the surface shows dynamic scaling at large lateral scales while conserving the ripple pattern at small scales. ${ }^{41}$ Since the transition to this regime is induced by the nonconserved $\mathrm{KPZ}$ terms, it is accompanied by a saturation of the ripple amplitude. ${ }^{24,41}$ In the present experimental results, however, coarsening seems to be observed even after the ripple amplitude has saturated (cf. Fig. 5). This again might indicate the relevance of the additional nonlinearities $\xi_{n, p}$, whose dynamical role has not been completely assessed (other than, e.g., their contribution to ripple motion with a nonuniform velocity), ${ }^{24}$ or perhaps the relevance of relaxation mechanisms other than those considered in two-fiel models. ${ }^{42}$

\section{CONCLUSION}

In summary, the evolution of Si surfaces during oblique high-fluenc ion sputtering was studied by ex situ AFM. The observed morphology consists of a well-ordered ripple pattern that is superposed by long-wavelength corrugations. These corrugations dominate the surface morphology at intermediate and high fluences Logarithmic coarsening of the ripple wavelength is observed. The dynamic scaling behavior at low fluence and short lateral scales indicates the presence of a KS type instability and a linear coefficien $0>\nu_{n} \gg \nu_{p}$ in the directions parallel and normal to the projection of the ion beam, respectively. At high fluences the large scale morphology of the surface exhibits scaling anisotropy with roughness exponents $\alpha_{n}=0.76 \pm 0.04$ and $\alpha_{p}=0.41 \pm 0.04$ in the $n$ and $p$ directions, respectively.

The observed algebraic scaling behavior at high fluence and large lateral scales does not agree with the anisotropic $\mathrm{KS}$ equation, which is expected to exhibit isotropic logarithmic scaling ${ }^{4,16}$ or rotated ripple structures ${ }^{17,19}$ under the current experimental conditions. However, while the presence of dispersive nonlinearities as in the general continuum equation of ion erosion, ${ }^{4}$ Eq. (9), might explain the experimentally observed strongly anisotropic scaling at the longest flu ences, the observed coarsening at intermediate fluence can be accounted for in principle by conserved-KPZ type nonlinearities. Thus we are naturally led to considering Eq. (10) as a potential continuum description of the present experiments. Indeed, such an equation has been derived for oblique incidence ion sputtering within the so-called two-fiel or "hydrodynamic" description. ${ }^{23,24}$ However, elucidating its scaling behavior will require future theoretical efforts. In particular, it remains to be seen whether the observation that ripple coarsening continues after the saturation of the ripple amplitude can be still accommodated by the dynamics of Eq. (10) or else it still requires further modification of the corresponding physical model.

\section{ACKNOWLEDGMENTS}

We thank M. Nicoli for discussions. This work was partially supported by MEC (Spain) through Grant No. FIS2006-12253-C06-01, by UC3M/CAM (Spain) through Grant No. CCG06-UC3M/ESP-0668, and by CAM (Spain) through Grant No. S-0505/ESP-0158. *a.keller@fzd.de

${ }^{1}$ M. Navez, C. Sella, and D. Chaperot, C. R. Hebd. Seances Acad. Sci. 254, 240 (1962).

${ }^{2}$ R. L. Cunningham, P. Haymann, C. Lecomte, W. J. Moore, and J. J. Trillat, J. Appl. Phys. 31, 839 (1960).

${ }^{3}$ E. Chason, T. M. Mayer, B. K. Kellerman, D. T. Mcllroy, and A. J. Howard, Phys. Rev. Lett. 72, 3040 (1994).

${ }^{4}$ M. A. Makeev, R. Cuerno, and A. L. Barabási, Nucl. Instrum. Methods Phys. Res. B 197, 185 (2002).

${ }^{5}$ J. Erlebacher, M. J. Aziz, E. Chason, M. B. Sinclair, and J. A. Floro, Phys. Rev. Lett. 82, 2330 (1999).

${ }^{6}$ C. C. Umbach, R. L. Headrick, and K. C. Chang, Phys. Rev. Lett. 87, 246104 (2001).

${ }^{7}$ S. Rusponi, G. Costantini, C. Boragno, and U. Valbusa, Phys. Rev. Lett. 81, 2735 (1998).

${ }^{8}$ D. P. Datta and T. K. Chini, Phys. Rev. B 69, 235313 (2004).

${ }^{9}$ V. K. Smirnov, D. S. Kibalov, O. M. Orlov, and V. V. Grabosh nikov, Nanotechnology 14, 709 (2003).

${ }^{10}$ M. O. Liedke, B. Liedke, A. Keller, B. Hillebrands, A. Mück lich, S. Facsko, and J. Fassbender, Phys. Rev. B 75, 220407(R) (2007).

${ }^{11}$ T. W. H. Oates, A. Keller, S. Facsko, and A. Mücklich, Plasmon ics 2, 47 (2007).
${ }^{12}$ R. M. Bradley and J. M. E. Harper, J. Vac. Sci. Technol. A 6, 2390 (1988)

${ }^{13}$ P. Sigmund, J. Mater. Sci. 8, 1545 (1973).

${ }^{14}$ M. A. Makeev and A. L. Barabási, Appl. Phys. Lett. 71, 2800 (1997).

${ }^{15}$ T. M. Mayer, E. Chason, and A. J. Howard, J. Appl. Phys. 76, 1633 (1994)

${ }^{16}$ R. Cuerno and A. L. Barabási, Phys. Rev. Lett. 74, 4746 (1995).

${ }^{17}$ S. Park, B. Kahng, H. Jeong, and A. L. Barabási, Phys. Rev. Lett. 83, 3486 (1999).

${ }^{18}$ J. Erlebacher, M. J. Aziz, E. Chason, M. B. Sinclair, and J. A. Floro, J. Vac. Sci. Technol. A 18, 115 (2000).

${ }^{19}$ M. Rost and J. Krug, Phys. Rev. Lett. 75, 3894 (1995).

${ }^{20}$ S. Habenicht, W. Bolse, K. P. Lieb, K. Reimann, and U. Geyer, Phys. Rev. B 60, R2200 (1999).

${ }^{21}$ B. Ziberi, F. Frost, T. Höche, and B. Rauschenbach, Phys. Rev. B 72, 235310 (2005).

${ }^{22}$ S. Facsko, T. Bobek, A. Stahl, H. Kurz, and T. Dekorsy, Phys. Rev. B 69, 153412 (2004).

${ }^{23}$ J. Muñoz García, M. Castro, and R. Cuerno, Phys. Rev. Lett. 96, 086101 (2006).

${ }^{24}$ J. Muñoz García, R. Cuerno, and M. Castro, Phys. Rev. B 78, 205408 (2008). 
${ }^{25}$ A. L. Barabási and H. E. Stanley, Fractal Concepts in Surface Growth (Cambridge University Press, London, 1995).

${ }^{26}$ T. Vicsek and F. Family, Phys. Rev. Lett. 52, 1669 (1984).

${ }^{27}$ R. Gago, L. Vázquez, R. Cuerno, M. Varela, C. Ballesteros, and J. M. Albella, Appl. Phys. Lett. 78, 3316 (2001).

${ }^{28}$ B. Schmittmann, G. Pruessner, and H. K. Janssen, Phys. Rev. E 73, 051603 (2006).

${ }^{29}$ W. Möller, W. Eckstein, and J. P. Biersack, Comput. Phys. Com mun. 51, 355 (1988).

${ }^{30}$ H. Gnaser, Low Energy Ion Irradiation of Solid Surfaces, 1 st ed. (Springer, New York, 1998).

${ }^{31}$ T. K. Chini, F. Okuyama, M. Tanemura, and K. Nordlund, Phys. Rev. B 67, 205403 (2003).

${ }^{32}$ R. Pastor Satorras and D. H. Rothman, J. Stat. Phys. 93, 477 (1998).

${ }^{33}$ A. Keller, S. Roßbach, S. Facsko, and W. Möller, Nanotechnol ogy 19, 135303 (2008).
${ }^{34}$ T. Bobek, S. Facsko, H. Kurz, T. Dekorsy, M. Xu, and C. Te ichert, Phys. Rev. B 68, 085324 (2003).

${ }^{35}$ D. P. Datta and T. K. Chini, Phys. Rev. B 76, 075323 (2007).

${ }^{36}$ G. Carter, J. Appl. Phys. 85, 455 (1999).

${ }^{37}$ R. Cuerno, H. A. Makse, S. Tomassone, S. T. Harrington, and H. E. Stanley, Phys. Rev. Lett. 75, 4464 (1995).

${ }^{38}$ M. Kardar, G. Parisi, and Y. C. Zhang, Phys. Rev. Lett. 56, 889 (1986).

${ }^{39}$ B. Davidovitch, M. J. Aziz, and M. P. Brenner, Phys. Rev. B 76, 205420 (2007).

${ }^{40}$ J. T. Drotar, Y. P. Zhao, T. M. Lu, and G. C. Wang, Phys. Rev. E 59, 177 (1999).

${ }^{41}$ J. Muñoz García, R. Cuerno, and M. Castro, Phys. Rev. E 74, 050103(R) (2006).

${ }^{42}$ J. Muñoz García, L. Vázquez, R. Cuerno, J. A. Sánchez García, M. Castro, and R. Gago, in Toward Functional Nanomaterials, edited by Z. M. Wang (Springer, New York, 2009). 